\title{
PERFORMANCE OF DAILY DISPOSABLE CONTACT LENSES IN SYMPTOMATIC WEARERS
}

Joachim Nick, Dipl. Ing. (FH) Augenoptik'; Stefan Schwarz, Dipl.-Ing. (FH), FAAO 2; Satu Jarvinen, BSOptom $^{3}$; Ian Chalmers, FBDO CL(HONS) ${ }^{4}$; Carolina Kunnen, BOptom, BHealth, $\mathrm{PhD}^{5}$

${ }^{1}$ Alcon Pharma GmbH, Grosswallstadt, Germany

${ }^{2}$ Optometrie Stefan Schwarz FAAO, Hildesheim, Germany

${ }^{3}$ Optikko Kirkassilmä Oy, Torikeskus, Forssa, Finland

${ }^{4}$ Chalmers Opticians, Albany Road, Cardiff, UK

${ }^{5}$ Alcon Research LLC, Fort Worth, TX, USA

Corresponding Author: carolina.kunnen@alcon.com

Submitted: August 26, 2019. Accepted: January 9, 2020. Published: February 5, 2020.

\section{ABSTRACT}

\section{Purpose}

To evaluate the performance of delefilcon A water gradient and narafilcon A silicone hydrogel daily disposable contact lenses (CLs) in symptomatic soft CL wearers.

\section{Methods}

This multicenter, open-label, crossover study randomized 121 soft CL wearers with symptoms of CL discomfort to delefilcon A or narafilcon A for 2 weeks, followed by the alternate lens for 2 weeks. Subjects rated end-of-day (EOD) comfort, EOD dryness, and quality of vision, and investigators rated fit, surface deposits, and surface wettability.

\section{Results}

After 2 weeks, all subjective measures were better for delefilcon A than for narafilcon A, including EOD comfort $(8.3 \pm 1.9$ vs. $6.6 \pm 2.2)$, EOD dryness $(8.0 \pm 2.2$ vs. $5.8 \pm 2.6)$, and quality of vision $(8.9 \pm 1.4$ vs. $7.9 \pm 1.7$ ), all $p<.0001$. Average daily wear time (DWT; $13.0 \pm 2.8$ vs. $12.3 \pm 2.6 \mathrm{hr}$ ) and average comfortable DWT (11.6 \pm 3.9 vs. $9.3 \pm 3.8 \mathrm{hr})$ were longer for delefilcon A lenses $(p<.0001)$. The proportions of right and left lenses without front surface deposits were twofold higher for delefilcon A than for narafilcon $\mathrm{A}$, and surface wettability was significantly higher for delefilcon A than for narafilcon A $p<.0001$ ).

J Cont Lens Res Sci Vol 4(1):e1-e11; February 5, 2020

This article is distributed under the terms of the Creative Commons Attribution-Non Commercial 4.0 International License.CKunnen, et al. 


\section{Conclusion}

In this population of symptomatic CL wearers, delefilcon A lenses showed superior subjective ratings for comfort, dryness, quality of vision, DWT, and comfortable DWT and better investigator-rated lens surface attributes including fewer surface deposits and superior wettability than did narafilcon A lenses after 2 weeks of wear.

Key Words: daily disposable contact lens; performance; delefilcon A; narafilcon A; comfort; surface deposits.

Daily disposable contact lenses accounted for more than one in four $(29 \%)$ contact lens fittings worldwide in 2017 and even higher proportions of fittings in Canada, the United Kingdom, some Northern European countries, and Japan, Taiwan, and Singapore. ${ }^{1}$ In the United States, the use of daily disposable contact lenses increased roughly fivefold from 2002 to 2014 . $^{2}$ In addition to offering increased convenience and enhancing compliance, daily disposable contact lenses have been found in some studies to provide superior comfort and vision compared with reusable lenses. ${ }^{3,4}$ In one study, habitual wearers of weekly or monthly lenses who were switched to daily disposable lenses showed improved contact lens-related symptoms and objective signs with the biomicroscope. ${ }^{5}$

The use of silicone hydrogel materials in contact lens fabrication has also risen markedly over the past two decades. Worldwide, more than one-half (56\%) of lenses fitted in 2017 were manufactured of silicone hydrogels materials, and in many countries, such as the United States, United Kingdom, and other Western European nations, most lenses fitted were silicone hydrogel. Of the daily disposable lenses fitted, one-half were silicone hydrogels. The introduction of silicone hydrogel materials in contact lenses was based, in large part, on greatly improved oxygen permeability. ${ }^{6,7}$ However, the siloxane monomers in silicone hydrogel lenses are relatively hydrophobic, raising concerns about wettability. ${ }^{7}$ In designing silicone hydrogel lenses, manufacturers have taken various approaches to overcome this challenge and improve wettability. ${ }^{7,8}$

These design methods notwithstanding, the investigation continues into how to optimize the wettability of silicone hydrogel lenses, to improve comfort and visual performance and reduce symptoms ${ }^{6-8}$ Across contact lens types and materials, the percentage of lens wearers who stop using their lenses remains high, as does the percentage of lens wearers identified as symptomatic, with ocular dryness and discomfort reported as the leading causes of contact lens discontinuation. ${ }^{9,10}$

Delefilcon A (DAILIES TOTAL $1{ }^{\circledR}$; Alcon Laboratories, Inc., Fort Worth, TX, USA) water gradient contact lenses contain a silicone-rich, highly breathable core (33\% water) and an ultra-soft hydrophilic surface gel ( $>80 \%$ water) to address the desired properties at the lens core and surface. ${ }^{11,12}$ Delefilcon A lenses provide high oxygen levels to the cornea, have shown low friction between their outer surface and the posterior eyelids, and have been associated with high patient-reported comfort levels. ${ }^{13-15}$

Short-term studies have compared delefilcon A with other soft daily disposable contact lenses in symptomatic and asymptomatic patients. A comparison of delefilcon A lenses with narafilcon A lens (1-DAY ACUVUE ${ }^{\circledR}$ TruEye ${ }^{\circledR}$; Johnson \& Johnson Vision Care, Inc., Jacksonville, FL) and somofilcon A lens (CLARITI $^{\circledR} 1$ Day; CooperVision, Inc., Pleasanton, CA) worn for 3 consecutive days each by contact lens wearers who were symptomatic or asymptomatic for end-of-day dryness found that delefilcon A lenses had significantly higher lens surface wettability, lower levels of visible front surface deposits after eight hours of wear, and longer pre-lens noninvasive tear breakup time than the two other lenses. ${ }^{16}$ Also, delefilcon A was superior to the other two lenses with respect to cumulative comfort, a measurement of comfort throughout the wearing interval that takes into account differences between wearing time. ${ }^{17}$

Longer-term studies may provide information not obtainable from short-term, 3-day evaluations. Expanding the study period from 3 days to 2 weeks may provide a greater opportunity to assess potential adverse events and obtain subjective feedback from study subjects. Also, longer-term studies can

J Cont Lens Res Sci Vol 4(1):e1-e11; February 5, 2020

This article is distributed under the terms of the Creative Commons Attribution-Non

Commercial 4.0 International License.CKunnen, et al. 
provide a greater number of opportunities to measure dryness, end-of-day dryness, lens comfort, and lens surface wettability, as well as allowing longitudinal determination of objective and subjective parameters over a longer period.

It was therefore of interest to further study the clinical performance of delefilcon A and narafilcon A lenses after longer periods of wear in contact lens wearers who experience dryness or other symptoms of lens-related discomfort. This randomized crossover study compared the performance of delefilcon A water gradient daily disposable lenses with narafilcon A daily disposable lenses in current symptomatic lens users who wore each lens type for 2 weeks.

\section{METHODS}

\section{Subjects}

The study was performed at eight sites in Europe (two in Finland, three in Germany, and three in the United Kingdom). The study was conducted according to the principles outlined in the Declaration of Helsinki and according to Good Clinical Practice guidelines. The study protocol was approved by the Ethics Committees at the relevant institutions, and all subjects provided written informed consent. To ensure observer consistency across sites, all sites used the same protocol, questionnaires, and grading scales.

Subjects were eligible for inclusion if they were aged 18 years or older, were current soft contact lens wearers and were identified as "symptomatic" at the baseline visit based on their responses to the three statements on the eligibility questionnaire (Table 1). Subjects were required to have a lens prescription with sphere powers in the range of $-0.50 \mathrm{D}$ to -10.00 $\mathrm{D}$, cylinder powers $\leq 0.75 \mathrm{D}$, and no ADD correction. They also had to achieve visual acuity of at least 6/7.5 meters (logMAR 0.10) in each eye with their habitual soft contact lenses and be willing to wear the study lenses at least 8 hours/day and at least 5 days per week.

Subjects were excluded if they were current wearers of delefilcon A or narafilcon A lenses, were neophyte contact lens users, had systemic or ocular disease or disorder (although refractive disorder was allowed and dry eye permitted), or had complicating factors or a structural abnormality that would have impaired their ability to wear lenses during the study. Also excluded were subjects unwilling to discontinue mechanical eyelid therapy, pregnant and nursing women, and individuals who had participated in other clinical trials within the previous 30 days.

At the baseline/screening visit (Visit 1), subjects who met the inclusion criteria were randomized (1:1) to wear either delefilcon A (lens diameter, $14.1 \mathrm{~mm}$; base curve, $8.5 \mathrm{~mm}$ ) or narafilcon A (lens diameter, $14.2 \mathrm{~mm}$; base curve, $8.5 \mathrm{~mm}$ or $9.0 \mathrm{~mm}$ ) lenses bilaterally for 2 weeks. After being fitted with the first set of lenses according to the manufacturers' guidelines, subjects were evaluated by the investigators for visual acuity,

TABLE 1 Eligibility Questionnaire

\begin{tabular}{|l|l|}
\hline \multicolumn{1}{|c|}{ Eligibility Questionnaire Statement } & \multicolumn{1}{|c|}{ Answer Conferring Eligibility $^{*}$} \\
\hline My contacts are comfortable all day long. & Strongly disagree OR Disagree \\
\hline \multicolumn{2}{|c|}{ OND } \\
\hline $\begin{array}{l}\text { During the day, I take my contacts out earlier than I like } \\
\text { because my eyes feel dry. }\end{array}$ & Agree OR Strongly agree \\
\hline \multicolumn{2}{|c|}{ OR } \\
\hline $\begin{array}{l}\text { Late in the day, my eyes become dry, but I continue to wear } \\
\text { my contacts. }\end{array}$ & Agree OR Strongly agree \\
\hline
\end{tabular}

*To be identified as symptomatic and eligible for study entry, subjects had to answer "Strongly disagree" or "Disagree" to the first statement and "Agree" or "Strongly agree" to either or both of the second and third statements.

Five possible responses to these questions were allowed: Strongly disagree, Disagree, Undecided, Agree and Strongly Agree. To be identified as symptomatic and eligible for study entry, subjects had to answer "Strongly disagree" or "Disagree" to the first statement and "Agree" or "Strongly agree" to either or both of the second and third statements Likert. ${ }^{25}$ 
centration, overall lens fit, lens surface characteristics, lens fit satisfaction, and surface wettability. At Visit 2 (14 \pm 3 days after Visit 1), subjects were evaluated for all endpoints and fitted with the alternative study lenses. Lens characteristics were evaluated at Visit 1. At Visit 3 (14 \pm 3 days after Visit 2), subjects were evaluated for all endpoints.

\section{Subject-Reported Outcomes}

Subjects rated their habitual lenses at baseline and after wearing each set of test lenses for 2 weeks using a 10 -point rating scale, where $1=$ poor and $10=$ excellent. Comfort ratings included end-of-day (EOD) comfort, lens comfort lasting throughout the day, and consistent lens comfort from day to day; vision ratings included quality of vision during the day and at night and day-to-day consistency of vision; and dryness ratings included reduced feeling of dryness throughout the day, at the end of the day, and when using a computer or digital device. Subjects were also asked to record the number of days per week they wore their lenses, their average daily wear time in hours per day, and their average comfortable daily wear time in hours per day. Subjects were allowed to use various marketed rewetting drops and rinsing solutions throughout the study, with the investigator being responsible for recording the type and frequency of each.

At the end of the study, subjects were also administered a different questionnaire asking, "Over the entire period of wearing these lenses, how often did your eyes feel dry at the end of the day?" (Never, Rarely, Sometimes, Frequently, or Constantly). Also, subjects were asked to state their preferences for the two lenses relative to eight criteria (Table 2).

\section{Investigator-Reported Outcomes}

Using a five-point system, investigators reported their satisfaction with lenses based on overall lens fit (Unacceptably tight, Acceptably tight, Optimal, Acceptably loose, or Unacceptably loose) and centration (Centered, Slightly decentered, Mildly decentered without limbal touch, Moderately decentered with limbal touch but without corneal exposure, and Severely decentered with corneal exposure). Based on biomicroscopy, investigators evaluated lens surface characteristics and front- and back-surface deposits and dry/non-wetting areas on scales of 0 to $4(0=$ None, $1=$ Very slight, $2=$ Slight, $3=$ Moderate, and $4=$ Severe $)$ and rated their overall impressions of surface wettability on a 10 -point rating scale $(1=$ poor to $10=$ excellent $)$ after each 2-week lens-wear period.

\section{Safety}

Adverse events were monitored throughout the study, beginning at the time of informed consent. Investigators performed biomicroscopy, rating limbal redness, bulbar redness, corneal epithelial edema, and stromal edema. Each of these was evaluated on a five-point scale, with $0=$ none, $1=$ trace, $2=$ mild, $3=$ moderate, and $4=$ severe. Also, corneal and conjunctival staining with fluorescein were performed, as was an evaluation for tarsal abnormalities and other

TABLE 2 Lens Comfort Attributes

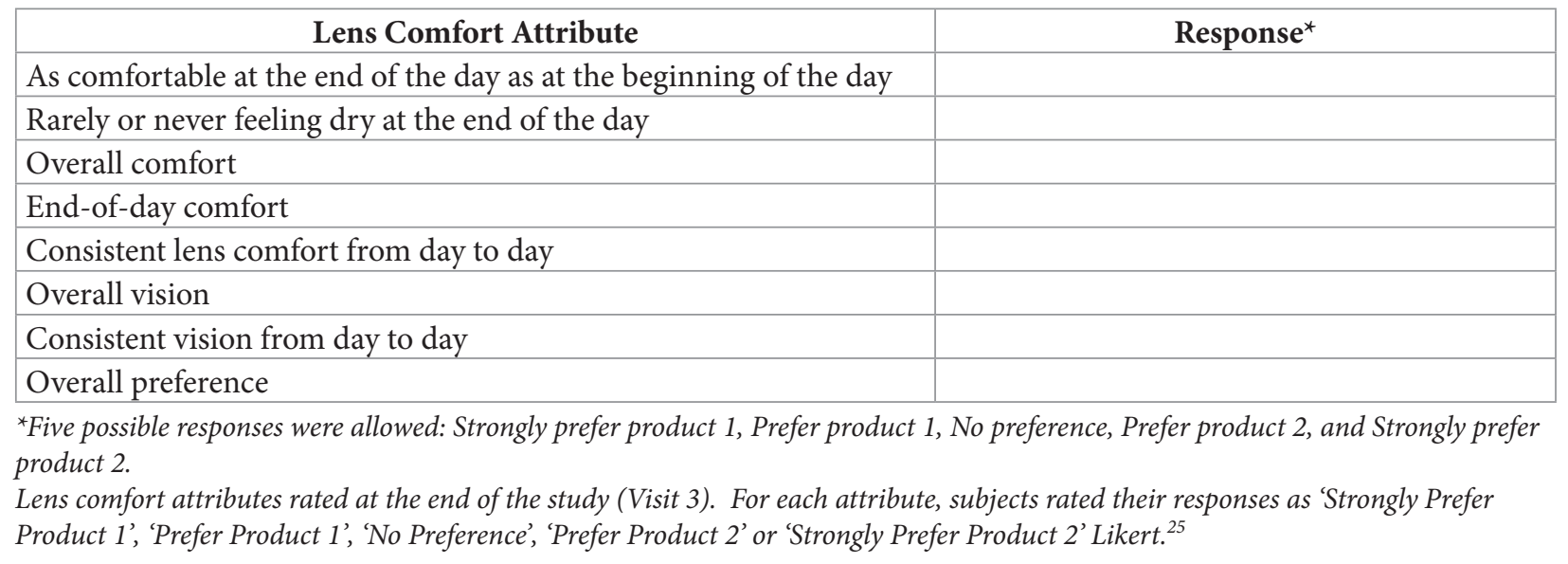

J Cont Lens Res Sci Vol 4(1):e1-e11; February 5, 2020

This article is distributed under the terms of the Creative Commons Attribution-Non Commercial 4.0 International License.CKunnen, et al. 
characteristics, with each of these rated on the same five-point scale. Adverse events were considered mild if the subject was aware of but could easily tolerate the sign or symptom; moderate if the sign or symptom resulted in discomfort significant enough to cause interference with the subject's usual activities; and severe if the sign or symptom was incapacitating and resulted in the subject's inability to work or engage in usual activities.

\section{Determination of Sample Size}

The sample size calculation was based on a similar study design comparing the performance of delefilcon A and narafilcon A lenses, each worn for three days. That study enrolled 50 subjects, each of whom wore the study lenses bilaterally and determined subjective ratings for each on a scale of $1-100$. In that study, the maximum standard deviation of the paired differences between the two lenses for end of day comfort was \pm 22 , with the corresponding averages for of delefilcon A and narafilcon A lenses being 74 and 70 , respectively. Converting linearly to a 1-10 rating scale resulted in a standard deviation of \pm 2.2 . Assuming a standard deviation of 2.2, a within-pair correlation of 0.5 , and a two-sided $\alpha$ of 0.05 , a minimum sample size of 96 would provide a $90 \%$ power to detect a superiority margin of one grade. Allowing for attrition and discontinuation, a paired study of 120 subjects would achieve the goal of 96 study completers.

\section{Statistical Methods}

The safety dataset consisted of all subjects enrolled and dispensed lenses, whereas the efficacy dataset consisted of all enrolled subjects who met the approval of the relevant Ethics Committee and completed the study. Subjective ratings of end-ofday comfort were assessed on efficacy subjects as a single measure on the continuous 1 to 10 rating scale during visits 2 and 3, which each occurring after the 2 weeks of product wear. A paired $t$-test was performed to assess treatment differences and a mixed-effect linear model was fitted to evaluate paired differences between test (delefilcon A) and control lenses (narafilcon A) for end-of-day comfort collected at the 2- week (Visit 2) and 4-week (Visit 3) visits. For all continuous variables, and ordinal variables expressed on a numerical scale, a continuous-variable summary ( $n$, mean, standard deviation, median, minimum, and maximum) was calculated for each measurement time point and for the changes between baseline and each post-baseline time point. For ordinal and categorical variables (including dichotomous variables), a categorical summary (frequency of response, percentage of response) was provided for the scores obtained at each assessment. Statistical analysis was performed by an independent biostatistician using SAS, release 9.1.3 or higher (SAS Institute, Cary, NC, U.S.A). All statistical tests were conducted at $\alpha=0.052$-sided unless otherwise specified.

\section{RESULTS}

\section{At Baseline, During Habitual Lens Wear}

A total of 123 contact lens wearers were screened, of whom 121 were randomized: 60 to delefilcon A lenses and 61 to narafilcon A lenses, followed by subsequent crossover to the other lens type (Figure 1). Three subjects did not complete the study and one was excluded because of premature enrollment. All three non-completers had been randomized to narafilcon A followed by delefilcon A lenses. Two discontinued treatment during wear of narafilcon A lenses, one because of a time/job conflict and the other because of conjunctivitis; whereas the other discontinued because of severe keratitis.

A total of 117 subjects were included in the efficacy analysis, including 69 wearers of habitual daily disposable contact lenses and 52 wearers of weekly or monthly replacement lens. The mean age of all study subjects was $34.0 \pm 10.4$ years and the majority were female $(70 \%)$. The mean age of the group of daily disposable lens wearers was slightly higher than that of weekly/monthly lens wearers $(36.4 \pm 10.9$ vs $30.8 \pm 8.8$ years) and the daily disposable group also included fewer women (64\% vs 79\%). Baseline ratings (mean $\pm \mathrm{SD}$ ) for comfort and vision for all randomized subjects were: end-of-day comfort, 3.9 \pm 1.9 ; lens comfort throughout the day, $6.6 \pm 1.7$; vision quality during the day, $8.0 \pm 1.4$; and vision quality at night, $6.3 \pm 2.3$. The average daily wear time at baseline (with habitual lenses) was $11.9 \pm 2.8$ hours, and the average comfortable daily wear time at baseline was $8.3 \pm 3.1$ hours. 
FIG. 1 Subject participation, disposition, and flow.

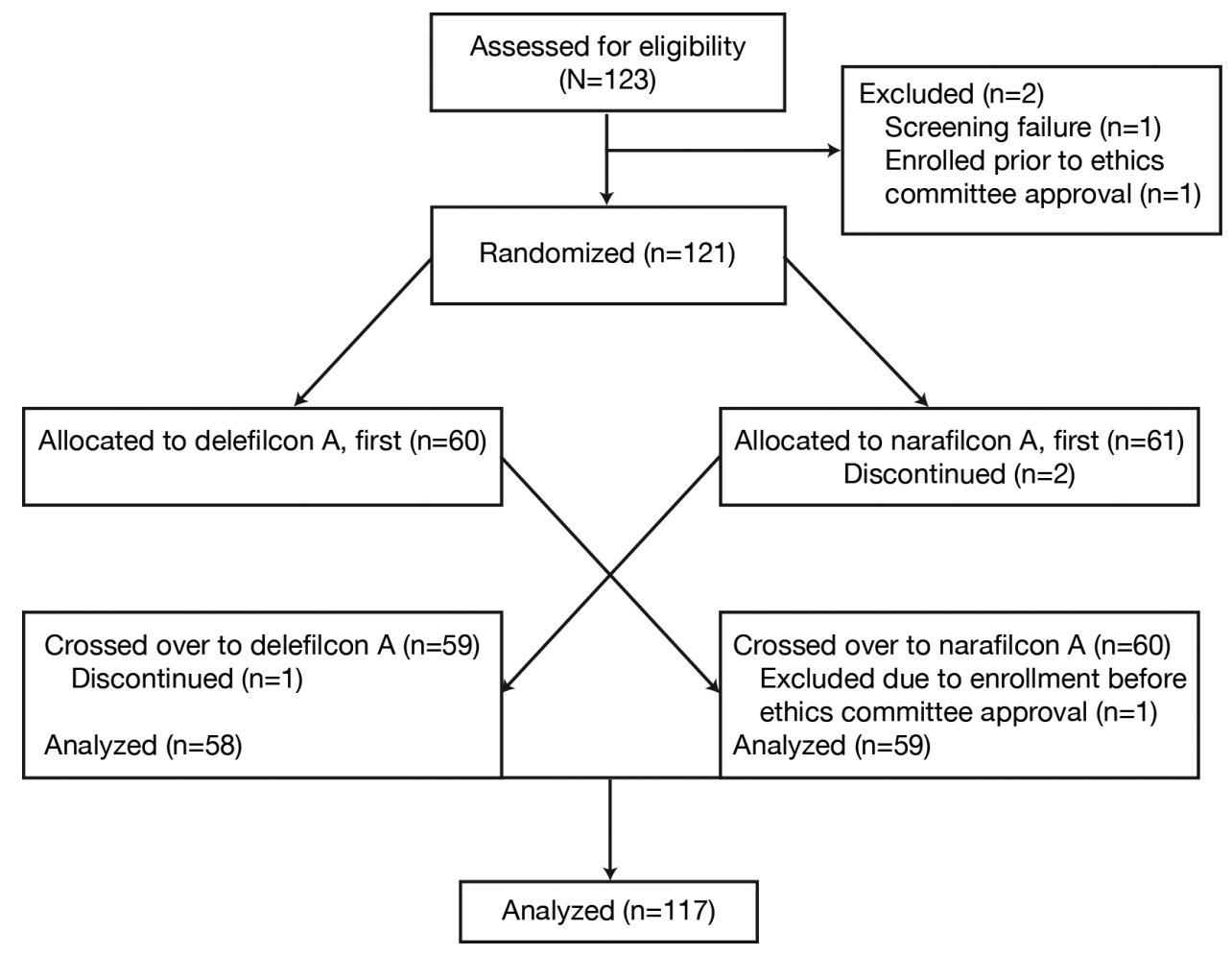

\section{Subject-Reported Outcomes During Study Lens Wear}

After 2 weeks of wearing the study lenses, endof-day comfort scores were significantly higher for delefilcon A than for narafilcon A lenses $(8.3 \pm 1.9$ vs $6.6 \pm 2.2$; least squares mean difference, $1.7 ; 95 \%$ confidence interval [CI], 1.2-2.2; $p<.0001$; Figure 2). Subject ratings of lens comfort throughout the day $(8.5 \pm 1.7$ vs. $7.0 \pm 2.1$; least squares mean difference, 1.6 ; $95 \%$ CI, 1.1-2.1; $p<.0001)$ and of consistent lens comfort from day to day $(8.6 \pm 1.8$ vs. $7.0 \pm 2.0$; least squares mean difference, 1.6 ; $95 \%$ CI, 1.1-2.1; $p<.0001)$ also were significantly higher for delefilcon A than for narafilcon A lenses. Subject ratings of quality of vision during the day $(8.9 \pm 1.4$ vs. $7.9 \pm 1.7$; least squares mean difference, $1.0 ; 95 \% \mathrm{CI}, 0.6-1.4$; $p<.0001)$, at night $(8.5 \pm 1.7$ vs. $7.4 \pm 1.8$; least squares mean difference, $1.1 ; 95 \% \mathrm{CI}, 0.7-1.5 ; p<.0001)$, and of consistent quality of vision from day to day $(8.8 \pm$ 1.6 vs $7.8 \pm 1.7$; least squares mean difference, 1.0 ; $95 \%$ CI, $0.6-1.4 ; p<.0001$ ) were also significantly higher for delefilcon A than narafilcon A lenses.
Subject ratings of feelings of dryness also favored delefilcon A lenses, including reduced feeling of endof-day dryness $(8.0 \pm 2.2$ vs. $5.8 \pm 2.6$; least squares mean difference, $2.2 ; 95 \%$ CI, 1.6-2.8; $p<.0001$; see Figure 2); reduced feeling of dryness throughout the day $(8.3 \pm 2.0$ vs. $6.4 \pm 2.5$; least squares mean difference, $1.8 ; 95 \% \mathrm{CI}, 1.3-2.4 ; p<.0001)$; and reduced feeling of dryness when using a computer, screen-based game, or hand-held device $(7.8 \pm 2.2 \mathrm{vs}$. $6.1 \pm 2.3$; least squares mean difference, $1.8 ; 95 \% \mathrm{CI}$, $1.2-2.4 ; p<.0001$ ), all of which were significantly higher for delefilcon A than for narafilcon A lenses.

Average daily wear time was significantly longer for delefilcon A than for narafilcon A lenses (13.0 \pm 2.8 vs. $12.3 \pm 2.6$ hours, $p=.001)$. Moreover, average comfortable daily wear time was more than 2 hours longer for delefilcon A than for narafilcon A (11.6 \pm 3.9 vs. $9.3 \pm 3.8$ hours, $p<.0001$ )

Subject responses to the questionnaire administered at the end of the study, after subjects had worn both lenses for 2 weeks each, regarding their lens preferences based on comfort and visual performance, including 
FIG. 2 Mean \pm SD patient-reported end-of-day (EOD) comfort, daytime quality of vision, nighttime quality of vision, and EOD dryness on a 10-point rating scale after 2 weeks of delefilcon A and narafilcon A lens wear. All, $\boldsymbol{p}<.0001$.

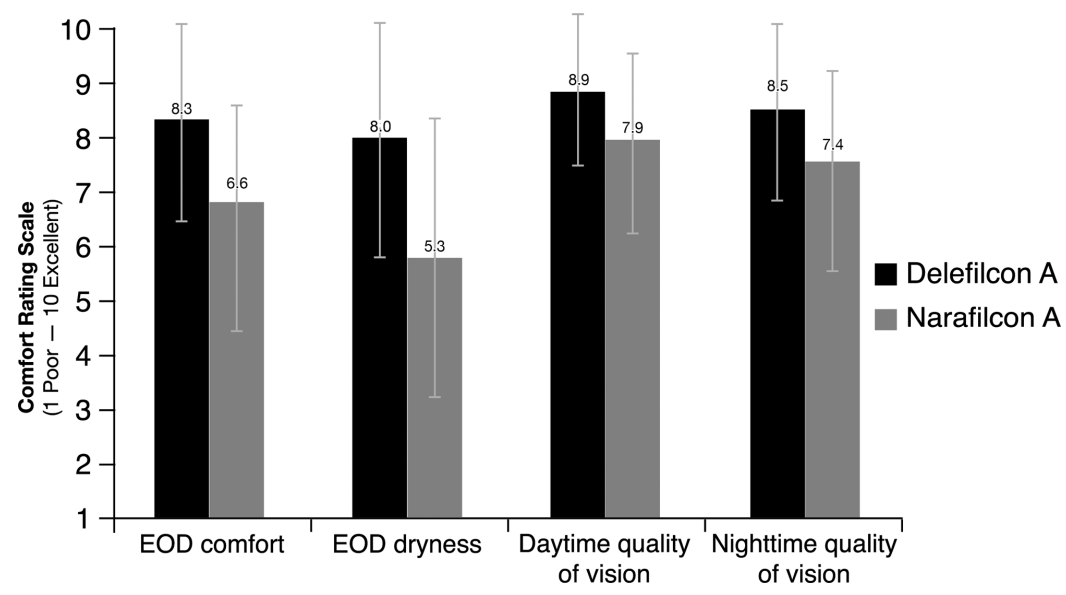

FIG. 3 Patient-reported preferences for delefilcon A or narafilcon A lenses after 2 weeks of wear in eight categories.

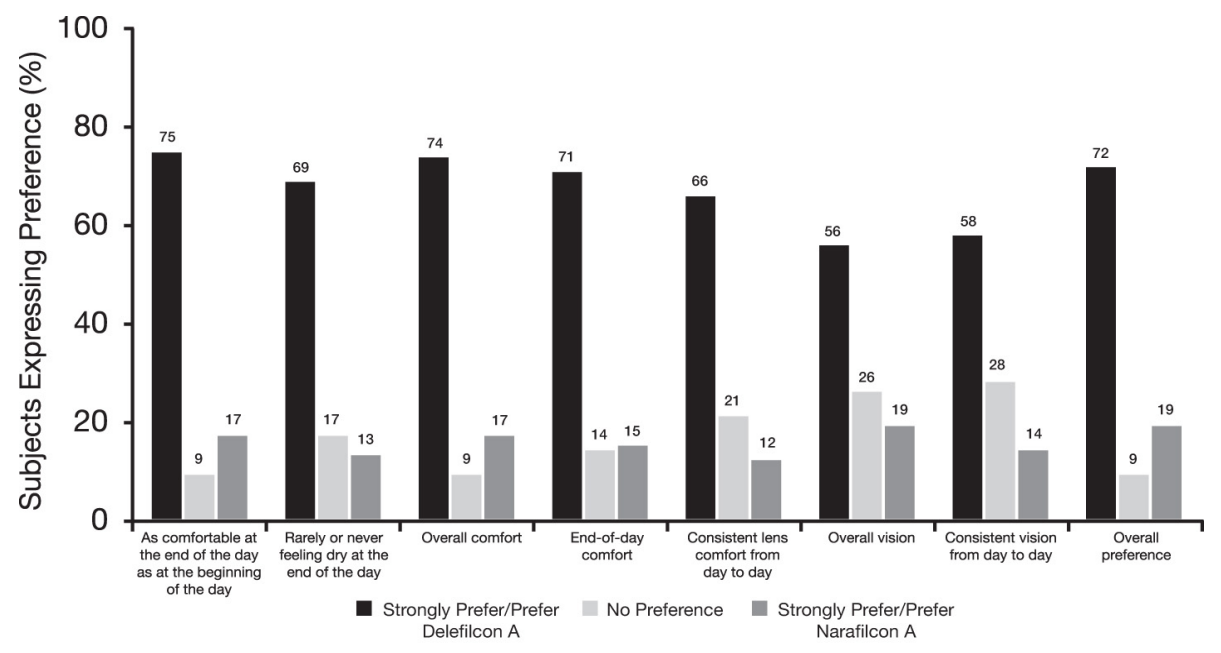

evaluations of end-of-day comfort, end-of-day dryness, and overall comfort, consistently showed subject preferences for delefilcon A over narafilcon A in all eight categories (Figure 3). For all comparisons, the preferences for delefilcon A lenses were statistically significant $(p<.0001$; Cochran-Mantel-Haenszel test).

\section{INVESTIGATOR-REPORTED OUTCOMES}

Results from investigator assessments of lens fit and centration showed better ratings for delefilcon A than for narafilcon A lenses. Overall lens fit was reported as 'Optimal' for $90.6 \%(212 / 234)$ of eyes fitted with delefilcon A lenses compared with 52.1\% (122/234) of eyes fitted with narafilcon A lenses (Figure 4). In assessments of centration, 94.4\% (221/234) of delefilcon A lenses were reported to be 'Centered,' with the remainder showing 'Slight decentration.' In comparison, $82.5 \%$ (193/234) of narafilcon A lenses were rated as 'Centered.'

Investigators' assessments of surface deposits and of wettability were also better with delefilcon A lenses than with narafilcon A. The proportions of lenses with 
FIG. 4 Investigator-reported percent of subjects with optimal lens fit and optimal lens centration for delefilcon $\mathrm{A}$ and narafilcon A lenses.

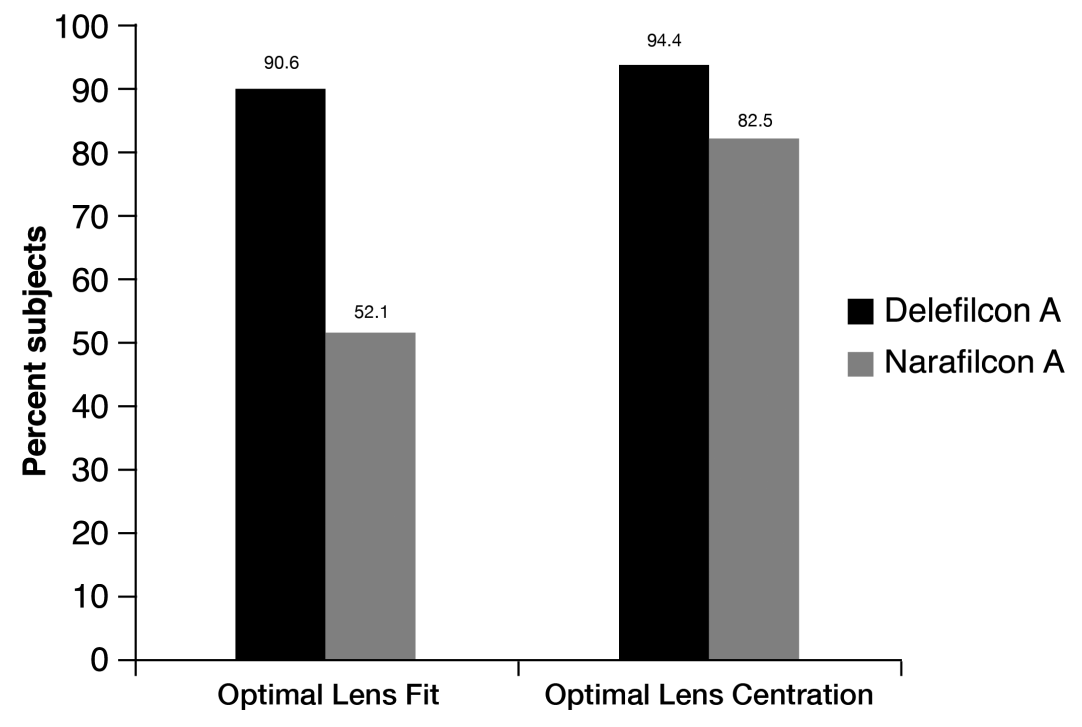

FIG. 5 Investigator-reported percent of wearers of delefilcon A and narafilcon A lenses with no visible front surface (FS) deposits, with no visible back surface (BS) deposits and with no dry/non-wetting areas.

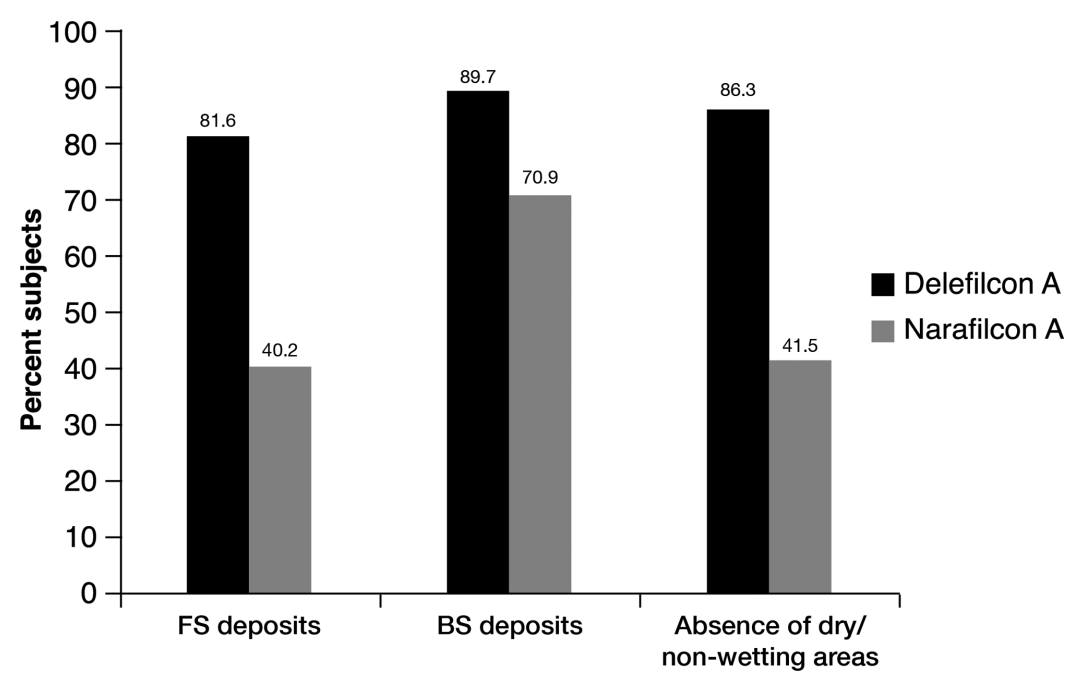

no visible front-surface deposits were approximately twofold higher for delefilcon A $(81.6 \%, 192 / 235)$ than for narafilcon A $(40.2 \%, 94 / 234)$ lenses (Figure 5). A higher percentage of delefilcon A lenses $(89.7 \%$, 210/234) than narafilcon A lenses $(70.9 \%, 166 / 234)$ had no visible back-surface deposits. Front- and back-surface deposits occurred less frequently on delefilcon A than on narafilcon A lenses; when present on delefilcon lenses, front-surface deposits were generally 'Very slight' (15\%), with some 'Slight' (2\%) or 'Moderate' $(1 \%)$ ratings. In contrast, front-surface deposits were present on the majority of narafilcon A lenses, although most were 'Very slight (50\%), with some 'Slight' ( $8 \%)$ or 'Moderate' (2\%). Back-surface deposits on delefilcon A lenses, when present, were "Very slight' $(10 \%)$, whereas back-surface deposits on 
narafilcon A lenses were generally "Very slight' (27\%), with some being 'Slight' (2\%) or 'Moderate' (1\%).

The proportion of lenses with no dry/non-wetting areas on their surfaces was also approximately twofold higher with delefilcon A $(86.3 \%, 202 / 234)$ than with narafilcon A (41.5\% 97/234) lenses (Figure 5). The investigators' overall impression of surface wettability after 2 weeks of lens wear was significantly better for delefilcon A than with narafilcon A lenses $(9.3 \pm 1.0$ vs $7.4 \pm 1.7, p<.0001$; Figure 6).

\section{Safety}

There were no serious adverse events reported during the study. Four adverse events were recorded, of which three were ocular, two with delefilcon A (one subject each with keratitis and ocular hyperemia), and one with narafilcon A (conjunctivitis). Ocular hyperemia and conjunctivitis were considered possibly related to treatment, whereas keratitis was not; rather, keratitis was deemed related to the disease under study (contact lens discomfort).

The most common abnormal post-baseline biomicroscopy findings were limbal redness, bulbar redness, corneal staining, and conjunctival staining, with fewer than $6 \%$ of subjects experiencing moderate outcomes for any characteristic in either eye. One subject experienced severe keratitis of the left eye, but this was considered by the investigator as unlikely to be related to the delefilcon A lens. No other serious adverse events were reported during the study.

\section{DISCUSSION}

This study evaluated subject-reported comfort levels in symptomatic contact lens wearers who wore delefilcon A and narafilcon A daily disposable silicone hydrogel lenses for two weeks each instead of their habitual contact lenses. To participate in the study, lens wearers had to answer 'Disagree' or 'Strongly disagree' to the statement regarding comfort and 'Agree' or 'Strongly agree' to one or both statements regarding contact lens-associated dryness with their habitual lenses. In assessing end-of-day comfort, subjects rated delefilcon A significantly higher than narafilcon A lenses. They also gave significantly better ratings for dryness throughout the day, dryness at the end of day, and dryness during use of a computer, screen-based game, or hand-held device with delefilcon A lens wear than with narafilcon A lens wear. Moreover, investigator-reported ratings were consistent with subjective ratings for the two lens types. According to investigator ratings, twice as many subjects were free of dry/non-wetting areas during delefilcon $\mathrm{A}$ lens wear than during narafilcon A wear, and overall assessments of surface wettability were significantly better for delefilcon A than for narafilcon A.

Contact lens comfort has been associated with lubricity, as indicated by a significant inverse correlation between comfort indices and coefficient of friction. ${ }^{18-20}$ The high comfort levels, even after

FIG. 6 Mean \pm SD investigator-reported average of lens surface wettability on a 10-point rating scale for delefilcon $\mathrm{A}$ and narafilcon $\mathrm{A}$ lenses.

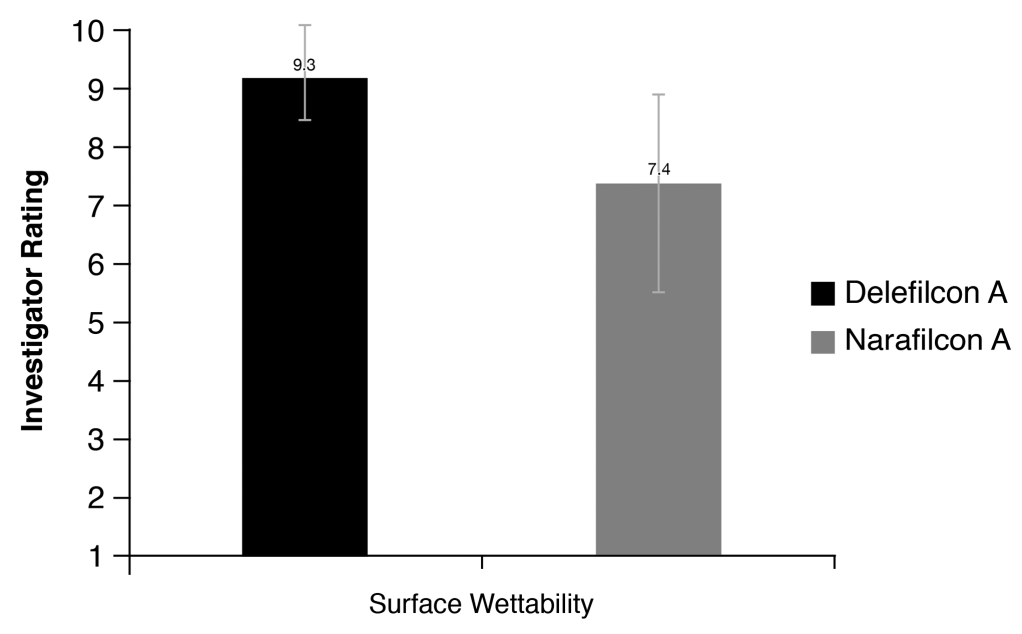

J Cont Lens Res Sci Vol 4(1):e1-e11; February 5, 2020

This article is distributed under the terms of the Creative Commons Attribution-Non

Commercial 4.0 International License.CKunnen, et al. 
prolonged wear, observed with delefilcon A in this study and previous investigations may result from the high lubricity of delefilcon A, which has been attributed to high surface water content resulting from their unique water gradient characteristics. In one study, the critical coefficient of friction and lubricity factor (1/critical coefficient of friction) of delefilcon A lenses were calculated and measured after 15 minutes and 16 hours of wear. After 16 hours of wear, the lubricity of delefilcon was shown to be equivalent to that of unworn (delefilcon A) lenses and equivalent to that of lenses worn for 15 minutes. In line with the previously published association between comfort and coefficient of friction, this maintenance in lubricity through the entire wear day corresponded with a high level of comfort during wear. ${ }^{21}$

Visible deposits on the contact lens surface have been correlated with reduced comfort levels, and the clearance of those deposits with increased comfort. ${ }^{5,22-24}$ In this study, the proportions of lenses without visible front-surface deposits were about twofold higher for delefilcon A than for narafilcon A, suggesting an association between greater wettability and deposits. This greater wettability could potentially reduce friction between the lens and the eyelid. Investigator-rated dry/non-wetting areas were twofold lower for delefilcon A than for narafilcon A lenses and surface wettability was significantly higher in delefilcon A lenses.

A limitation of this randomized crossover study design is that both subjects and investigators were unmasked as to lens type. Another limitation was the lack of a wash-out period between the wearing of the first lens and wearing of the second lens.

In conclusion, both daily disposable contact lenses performed well, with delefilcon A lenses showing superior subjective outcome ratings compared with narafilcon A lenses after 2 weeks of wear. As indicated by investigator ratings, delefilcon A lenses also had better lens surface attributes in this symptomatic contact lens wearing population. The superior subjective outcome ratings associated with better lens surface attributes after two weeks suggests that delefilcon A lenses are a good selection for symptomatic contact lens wearers.

\section{DISCLOSURES}

Portions of this study were presented at the Contact Lens Association of Ophthalmologists International Symposium \& Congress, Toronto, Ontario, Canada, June 12-14, 2014 and at the American Academy of Optometry 2014 Annual Meeting, Denver, Colorado, November 12-15, 2014.

This study was funded by Alcon Research, Ltd. $\mathrm{JN}$ and $\mathrm{CK}$ are employees of Alcon. Editorial support was provided by BelMed Professional Resources, Inc., which was contracted and funded by Alcon.

\section{ClinicalTrials.Gov ID: $\quad$ NCT01561560}

\section{REFERENCES}

1. Morgan PB, Woods CA, Tranoudis IG, et al. International contact lens prescribing in 2017. Contact Lens Spectrum 2018;33(1):28-33.

2. Efron N, Nichols JJ, Woods CA, Morgan PB. Trends in US contact lens prescribing 2002 to 2014. Optom Vis Sci 2015;92(7):758-67.

3. Cho P, Boost MV. Daily disposable lenses: the better alternative. Cont Lens Anterior Eye 2013;36(1):4-12.

4. Ichijima $\mathrm{H}^{1}$, Karino $\mathrm{S}$, Sakata H, Cavanagh HD. Improvement of subjective symptoms and eye complications when changing from 2-week frequent replacement to daily disposable contact lenses in a subscriber membership system. Eye Contact Lens 2016;42(3):190-5

5. Fahmy M, Long B, Giles T, Wang CH. Comfort-enhanced daily disposable contact lens reduces symptoms among weekly/monthly wear patients. Eye Contact Lens 2010;36(4):215-9.

6. Jones L, Brennan NA, Gonzalez-Meijome J, et al; members of the TFOS International Workshop on Contact Lens Discomfort. The TFOS International Workshop on Contact Lens Discomfort: report of the contact lens materials, design, and care subcommittee. Invest Ophthalmol Vis Sci 2013;54(11):TFOS37-70.

7. Keir N, Jones L. Wettability and silicone hydrogel lenses: a review. Eye Contact Lens 2013;39(1):100-8.

8. Stapleton F, Tan J. Impact of contact lens material, design, and fitting on discomfort. Eye Contact Lens 2017;43(1):32-9.

9. Dumbleton K, Woods CA, Jones LW, Fonn D. The impact of contemporary contact lenses on contact lens discontinuation. Eye Contact Lens 2013;39(1):93-9. 
10. Dumbleton K, Caffery B, Dogru M, et al; members of the TFOS International Workshop on Contact Lens Discomfort. The TFOS International Workshop on Contact Lens Discomfort: report of the subcommittee on epidemiology. Invest Ophthalmol Vis Sci 2013;54(11):TFOS20-36.

11. DAILIES TOTAL1 [package insert]. Fort Worth, TX: Alcon Laboratories, Inc; 2016.

12. Szczesna-Iskander DH. Comparison of tear film surface quality measured in vivo on water gradient silicone hydrogel and hydrogel contact lenses. Eye Contact Lens. 2014;40(1):23-27.

13. Michaud L, Forcier P. Comparing two different daily disposable lenses for improving discomfort related to contact lens wear. Cont Lens Anterior Eye 2016;39(3):203-9.

14. Insua Pereira F, Lira M. Comfort, ocular dryness, and equilibrium water content changes of daily disposable contact lenses. Eye Contact Lens 2018;44 Suppl 2:S233-40.

15. Marx S, Lauenborg B, Kern JR. Performance evaluation of delefilcon A water gradient daily disposable contact lenses in first-time contact lens wearers. Cont Lens Anterior Eye 2018;41(4):335-41.

16. Varikooty J, Schulze MM, Dumbleton K, et al. Clinical performance of three silicone hydrogel daily disposable lenses. Optom Vis Sci 2015;92(3):301-11.

17. Varikooty J, Keir N, Richter D, et al. Comfort response of three silicone hydrogel daily disposable contact lenses. Optom Vis Sci 2013;90(9):945-53.
18. Sterner O, Aeschlimann R, Zürcher S, et al. Friction measurements on contact lenses in a physiologically relevant environment: effect of testing conditions on friction. Invest Ophthalmol Vis Sci 2016;57(13):5383-92.

19. Vidal-Rohr M, Wolffsohn JS, Davies LN, Cerviño A. Effect of contact lens surface properties on comfort, tear stability and ocular physiology. Cont Lens Anterior Eye 2018;41(1):117-21.

20. Lau C, Tosatti S, Mundorf M, et al. Comparison of the lubricity and surface roughness of 5 cosmetic contact lenses. Eye Contact Lens 2018;44 Supp1 2: S256-65.

21. Maissa C, Nelson J, DeCenzo-Verbeten T, et al. Evaluation of the lubricity of Dailies Totall contact lenses after wear. Optom Vis Sci 2014;91(8):e-abstract 145195.

22. Campbell R, Kame G, Leach N, et al. Clinical benefits of a new multipurpose disinfecting solution in silicone hydrogel and soft contact lens users. Eye Contact Lens 2012;38(2):93-101.

23. Young G, Keir N, Hunt C, Woods CA. Clinical evaluation of long-term users of two contact lens care preservative systems. Eye Contact Lens 2009;35(2):50-8.

24. Lievens CW, Kannarr S, Zoota L, Lemp J. Lid papillae improvement with hydrogen peroxide lens care solution use. Optom Vis Sci 2016;93(8):933-42.

25. Likert R. A technique for the measurement of attitudes. Arch Psychology 1932;140:1-55. 